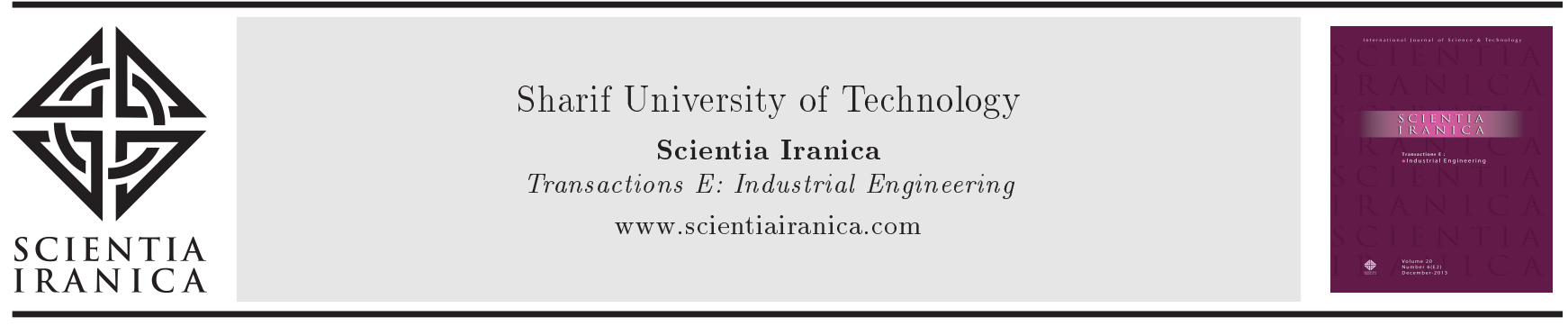

\title{
Optimization of weighted correlated multiple responses using a probabilistic index
}

\author{
M. Bashiri* and M.H. Bakhtiarifar \\ Department of Industrial Engineering, Faculty of Engineering, Shahed University, Tehran, Iran.
}

Received 25 September 2014; received in revised form 11 May 2015; accepted 15 June 2015

\section{KEYWORDS}

Cardinal weight; Ordinal weight; Multiple-response optimization; Correlation;

Transformation.

\begin{abstract}
Dealing with more than one response in the process optimization has been a great issue in recent years; therefore, multiple-response optimization studies have grown in the published works. In the common problems, there are some input variables which can affect output responses, but optimization can be more complex and more real when the responses have correlation with each other. In such problems, the analyst should consider the correlation structure in addition to the effects of input variables. In some cases, response variables may emerge by different distributions from the normal ones, which can be analyzed by the proposed method. Moreover, in some problems, response variables may have different levels of importance for the decision maker. In this study, we try to propose an efficient method to find the best treatment in an experimental design, which has different weights for correlated responses, either cardinal or ordinal. Also, a heuristic method is proposed to deal with problems that have a considerable number of correlated responses, or treatments. The results of some numerical examples confirm the validity of the proposed method. Moreover, a real case of air pollution in Tehran is studied to show the applicability of the proposed method in the real problems.
\end{abstract}

(C) 2016 Sharif University of Technology. All rights reserved.

\section{Introduction}

In recent years, due to the growth of industry and need to consider more than one output in the experiments, multiple responses optimization has been discussed in many articles. Accordingly, several methods have been proposed to optimize problems with multiple quality characteristics. Each method has its properties and the analyst should choose the best approach with regard to the problem issue. In some problems, all the responses have the same value for us and finding the best treatment will not be a very difficult problem. On the other hand, there are so many multiple-response problems with different levels of response importance in the real world cases. In these problems, a response

\footnotetext{
*. Corresponding author. Tel.: +98 21 51212092;

Fax: +982151212020

E-mail address: Bashiri.m@gmail.com (M. Bashiri)
}

may be more important for the analyst than others and the analyzer may be interested in finding the values of controllable factors with the least deviation from the more important response target.

By study of the literature, it can be seen that there are many methods to optimize multiple-response problems when each response has a weight. But, in most of them, the correlation between responses have been ignored. For example, Jeyapaul et al. [1] used the genetic algorithm to obtain weights for each response and optimized them. However, they assumed that responses were independent from each other. Chiao and Hamada [2] proposed a probabilistic method to study problems with correlated responses, but there is a question about the interpolating weights of each response in such method. Maghsoodloo and Chang [3] developed the quadratic loss function and signal to noise ratio for a bivariate response when both quality characteristics were from the same type. They interpolated social 
quality loss for each response to consider the importance of each quality characteristic. Maghsoodloo and Huang [4] focused on mixed bivariate responses and developed quadratic loss function and signal to noise ratios for them. Ozdemir and Maghsoodloo [5] extended quadratic quality loss function and signal to noise ratios for trivariate cases. Wu [6] proposed a method by considering the double-exponential desirability function, which has been modified based on Taguchi's loss function, to find the optimum values for correlated multiple quality characteristics. Ko et al. [7] suggested a new loss function method to accommodate robustness, quality of predictions, and bias in a single framework. Importance of each response was not considered in this approach. Some studies used Principal Component Analysis (PCA) to transform correlated responses to independent ones and then performed optimization. Antony [8] used only the first PC, but Liao [9] considered all PC's and proposed weighted principal component method. Datta et al. [10] performed PCA method and then utilized the genetic algorithm. Hejazi et al. [11] applied goal programming to model and optimize multi-response surfaces. They used group decision making to obtain the weights of response variables. In many publications, artificial neural network has been used to estimate values of responses for nonperformed treatments. For example, Noorossana et al. [12] used an artificial neural network to estimate the quantitative and qualitative response functions and then performed the optimization step by using genetic algorithm. Salmasnia et al. [13] used neurofuzzy and PCA to make a desirability function to optimize correlated multiple responses. Bashiri and Bakhtiarifar [14] proposed an optimality probability index to choose the best treatment in an experimental design with correlated normal responses. With regard to this information, it can be concluded that there is lack of an appropriate and practical method which can consider both cardinal and ordinal importance of correlation structure and responses.

Dealing with non-normal multivariate responses can be very problematic for most researchers. To simplify these problems for analysis, a transformation can be applied to normalize non-normal responses. Niaki and Abbasi [15] transformed multi-attribute data in a way that their marginal probability distributions had almost zero skewness. Riani [16] introduced alternative tests which did not require the maximum likelihood estimates of the transformation parameters.

Review of previous works shows that a comprehensive study on the multiple correlated non-normal responses optimization with different response weights does not exist; therefore, this study attempts to propose a method to consider the mentioned problem. To show the efficiency of the proposed method in real cases, an example about the air pollution problem of Tehran is studied.

The next section contains the proposed method, which starts with model statement in three cases of responses without weights, cardinally weighted responses, and ordinally weighted responses for normal and non-normal ones. Then, estimation of the needed parameters is explained in Section 3 and afterwards, a heuristic algorithm is presented to find the optimum treatment using the proposed indices in Section 4 section. In Section 5, some numerical examples of different cases and a real problem are given for better illustration of the proposed approach. Finally, the last section includes the conclusion and future research directions.

\section{Proposed method}

The aim of this method is to extend the optimality probability index, which has previously been proposed by Bashiri and Bakhtiarifar [14]. The presented index tries to find the best treatment by calculating its optimality probability in comparison with other treatments. In their proposed index, it is assumed that all responses are normally distributed while there is no a priori information of responses. In real cases, we deal with non-normal responses while some of them are more important than others; therefore, in this study, the optimality index is extended to be calculated in more real situations. The proposed method is explained for both normal and non-normal cases in three states of response importance. In a nutshell, the contributions of this paper, compared to Bashiri and Bakhtiarifar [14], can be mentioned as the ability of incorporating cardinal and ordinal weights as well as considering non-normal distribution for correlated responses.

\subsection{Normally distributed responses}

Suppose that we have an experimental design with $n$ treatments and $m$ normally distributed correlated responses. We interest to find the best treatment to achieve optimum values for the responses. To do this, we define a multivariate probability for each combination which shows the probability of being optimum in all responses between all the treatments. As mentioned before, the proposed method is considered in three states of non-weighted, cardinally weighted, and ordinally weighted of responses, which are described in the following subsections.

Each response can be of a Smaller The Better (STB), Larger The Better (LTB), or Nominal The Best (NTB) type. In the case of STB responses, it is obvious that a treatment which has smaller values can dominate another one with larger values of responses. We can state this by defining a probability measure as stated 
below:

$$
P I_{k, i}=P\left(y_{1 k}<y_{1 i}, \ldots, y_{m k}<y_{m i}\right),
$$

where $P I_{k, i}$ is the probability that $k$ th treatment is better than $i$ th treatment and $y_{m i}$ is the $m$ th response value corresponding to the $i$ th treatment.

To find the best treatment, the analyzer should find the Optimality Probability Index (OPI) proposed by Bashiri and Bakhtiarifar [14] for each treatment as follows:

$$
\mathrm{OPI}_{k}=\prod_{\substack{i=1 \\ i \neq k}}^{n} P\left(y_{1 k}<y_{1 i}, \ldots, y_{m k}<y_{m i}\right),
$$

where $\mathrm{OPI}_{k}$ is the optimality probability index of $k$ th treatment.

Similarly, we can find OPI for problems with LTB-type responses. In such problems, the best treatment should have larger response values than other treatments. In the case of NTB responses, we need to find absolute target corrected values for each response in each treatment. The treatment with the minimum absolute deviation value from its target is our favorite one. The OPI for the $k$ th treatment is product of probabilities that shows $k$ is better than other treatments:

$$
\begin{aligned}
\mathrm{OPI}_{k}= & \prod_{\substack{i=1 \\
i \neq k}}^{n} P\left(\left|y_{1 k}-t_{1}\right|<\left|y_{1 i}-t_{1}\right|, \ldots,\left|y_{m k}-t_{m}\right|\right. \\
& \left.<\left|y_{m i}-t_{m}\right|\right)
\end{aligned}
$$

where $y_{m i}$ is the $m$ th response corresponding to the $i$ th treatment and $t_{m}$ is target value for the $m$ th response when its type is Nominal The Best (NTB).

The multivariate probability function of absolute deviation for NTB responses can have another distribution rather than the normal one. So, calculating Eq. (3) may be very difficult. To solve this problem, we can transform NTB responses into STB-type by using the deviation terms as new variables. This transformation can violate the normality assumption. So, we should use another transformation on new STB values to change their distribution into the multivariate normal one.

In some cases, when we deal with some responses with different types, for example when the problem has some LTB and STB responses, we can use negative values for LTB-type response(s) to transform them into STB or use a mixed multivariate probability which can be obtained by changing Eq. (2).

\subsubsection{Multiple responses without weights}

In case of responses with the same importance, the proposed Bashiri and Bakhtiarifar's OPI [14] can be used. Moreover, other methods from literature, such as Chiao and Hamada [2], can be utilized as well.

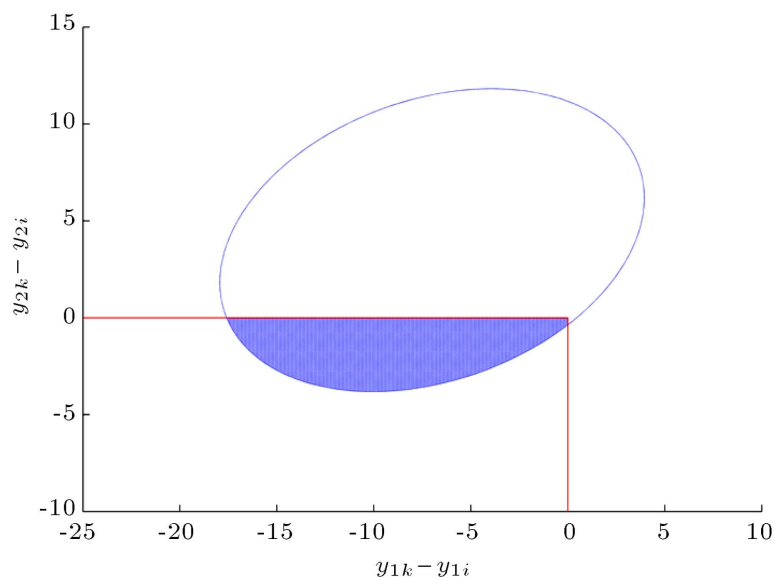

Figure 1. The confidence region for the mentioned probability.

\subsubsection{Multiple responses with cardinal weights}

In some problems, responses have different levels of importance for the analyzer. It means that one or more responses may be more attractive than the others. In these cases, we cannot use the previous equations to find the best treatment, because the importance of responses has been ignored in the OPI index. So, weights of responses should be considered in a new developed optimality index.

For better comprehension, describing an example can be helpful. Suppose that there is an experimental design with two correlated normal STB-type responses. The mean array of the responses for the $k$ th treatment is computed as [3 5], while it is equal to [10 1] for the $i$ th treatment. The covariance matrix of the responses in both of the treatments is equal to $\left[\begin{array}{cc}10 & 2 \\ 2 & 5.1\end{array}\right]$. Figure 1 illustrates the $95 \%$ confidence region for $P\left(y_{1 k}<\right.$ $\left.y_{1 i}, y_{2 k}<y_{2 i}\right)$.

It can be concluded that the $k$ th treatment has better value in the first response, but worse in the second one. In this situation, we expect that allocating greater weight to the first response would increase the desired probability while allocating greater weight to the second response would decrease it. At first, we transform the weights through dividing them by the minimum weight. Then, we perform a $t$-test for each response. If the test shows that treatment $k$ has better value in the $n$th response, then we multiple $y_{n i}$ by the corresponding transformed weight; but if the test shows that treatment $k$ has worse value in the $n$th response, then we multiple $y_{n k}$ by the corresponding transformed weight. Therefore, in the mentioned example, the weighted probability for treatment $k$ to be better than treatment $i ; \mathrm{WPI}_{k, i}$ is calculated as follows:

$$
\mathrm{WPI}_{k, i}=P\left(y_{1 k}<w_{1} y_{1 i}, w_{2} y_{2 k}<y_{2 i}\right) \text {, }
$$

where $w_{j}$ is the transformed weight of the $j$ th response. It is obvious that $\mathrm{PI}_{k, i}$ is a special version of $\mathrm{WPI}_{k, i}$ for 


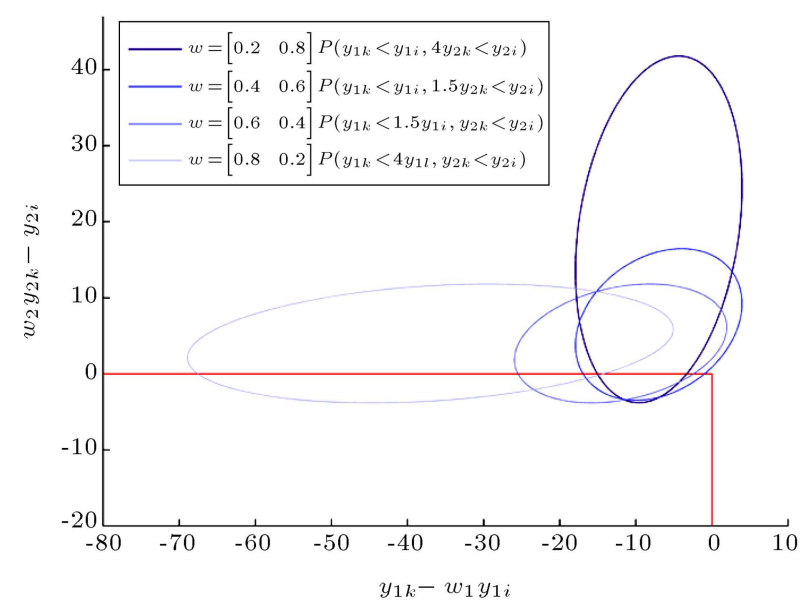

Figure 2. The confidence regions for the $\mathrm{WPI}_{k i}$ based on different weights.

equal weights. Figure 2 shows various $95 \%$ confidence regions based on different weights for $\mathrm{WPI}_{k, i}$ in the mentioned example.

In the case of LTB and NTB responses $\mathrm{WPI}_{k, i}$ is obtained by the same logic. Therefore, the Weighted Optimality Probability Index (WOPI) for the $k$ th treatment can be computed by multiplying WPIs as follows:

$$
\mathrm{WOPI}_{k}=\prod_{\substack{i=1 \\ i \neq k}}^{n} \mathrm{WPI}_{k, i} \text {. }
$$

\subsubsection{Multiple responses with ordinal weights}

In case of ordinal weights, we deal with responses which have different levels of importance defined by ranks. It means that order of the responses shows the importance of them. It is clear that in such cases, we do not have cardinal weight value for each response to find weighted optimality probability like the aforementioned equations. In this situation, we should consider responses ranking in the optimality probability calculation. For better comprehension, suppose that all of the responses are of the STB type. First, we sort responses based on their importance in a descending order and put them in a set named $J$. Then, to find the Ordinal Optimality Probability Index (OOPI) for the $k$ th treatment, we should calculate the probability of the $k$ th treatment to have a smaller value for $J(1)$ response as the most important response versus other possible treatments. If the most important response $(J(1))$ value for the $k$ th treatment is statistically equal to its value in the $i$ th treatment, then we should find the probability for the $k$ th treatment to have a smaller value of $J(2)$ than that of the $i$ th treatment, and so on. Therefore, to find OOPI, we need to perform a $t$-test to verify the hypothesis of equality of $J(1)$ values for the treatment and other ones. If the equality cannot be rejected, then another hypothesis test should be done on $J(2)$ value, and so on. After selecting the proper response based on this method, the effect of other responses from the measure should be eliminated. To do this, we find proper weight coefficients, $w_{j}$, for other responses such that the equality $t$-test on them in two compared treatments cannot be rejected and it has an enough great $p$-value. Note that the placing side of weight coefficients should be selected based on the explanations in the previous subsection. For better understanding, consider the example with two STB correlated responses in the previous subsection. The hypothesis $t$-test on the equality of $y_{1 k}$ and $Y y 1 i$ is rejected by a near zero $p$ value. Therefore, we should find a proper weight for the second response to eliminate its effect in the proposed measure as follows:

$$
\mathrm{OP}_{k, i}=P\left(y_{1 k}<y_{1 i}, w_{2} y_{2 k}<y_{2 i}\right) \text {. }
$$

By iterating these steps for all other treatments, the $\mathrm{OOPI}_{k}$ can be calculated as follows:

$$
\mathrm{OOPI}_{k}=\prod_{\substack{i=1 \\ i \neq k}}^{n} \mathrm{OP}_{k, i} .
$$

\subsection{Other multivariate distributions}

Suppose that the response values have another multivariate distribution rather than the normal one. In such problems, an idea is using a transformation to normalize responses before optimization stage. In this paper, NORTA inverse method, proposed by Niaki and Abbasi [17], is used to transform correlated values into normal ones. For better comprehension, consider normal to anything (NORTA) method (Cario and Nelson [18]) which can generate a $k$-dimensional random vector $\mathbf{X}$ where $X_{i}$ has an arbitrary cumulative distribution function $F_{X_{i}}$ with correlation matrix. In this situation, a transformation on a $k$-dimensional standard multivariate normal vector $\mathbf{Z}$ with correlation matrix $\sum_{z}$ should be performed to generate vector $\mathbf{X}$ as follows:

$$
\mathbf{X}=\left(\begin{array}{c}
F_{X_{1}}^{-1}\left[\Phi\left(z_{1}\right)\right] \\
F_{X_{2}}^{-1}\left[\Phi\left(z_{2}\right)\right] \\
\vdots \\
F_{X_{k}}^{-1}\left[\Phi\left(z_{k}\right)\right]
\end{array}\right),
$$

where $\Phi$ is the cumulative distribution function of a univariate standard normal. To find the correlation between $X_{i}$ and $X_{j}$, the following equation should be solved for each pair of variables:

$$
\begin{aligned}
E\left(X_{i} X_{j}\right) & =E\left(F_{X_{i}}^{-1}\left[\Phi\left(z_{i}\right)\right] F_{X_{j}}^{-1}\left[\Phi\left(z_{j}\right)\right]\right) \\
& =\int_{-\infty}^{+\infty} \int_{-\infty}^{+\infty} F_{X_{i}}^{-1}\left[\Phi\left(z_{i}\right)\right] F_{X_{j}}^{-1}\left[\Phi\left(z_{j}\right)\right] \\
& \varphi_{\rho_{z}(i, j)}\left(z_{i}, z_{j}\right) d z_{i} d z_{j} .
\end{aligned}
$$


Since these equations are usually unsolvable for many marginal distributions, Cario and Nelson [18] presented some theorems to help researchers do this. As another alternative, the simulation can be used to estimate the covariance matrix.

By such descriptions, as can be deduced, the NORTA inverse method transforms a vector of multiattribute variables such that they have multivariate normal distribution. The following formula is used for this purpose:

$$
\begin{aligned}
Y= & {\left[Y_{1}, Y_{2}, \ldots, Y_{k}\right]^{T}=\left[\Phi^{-1}\left(F_{X_{1}}\left(x_{1}\right)\right),\right.} \\
& \left.\Phi^{-1}\left(F_{X_{2}}\left(x_{2}\right)\right), \ldots, \Phi^{-1}\left(F_{X_{k}}\left(x_{k}\right)\right)\right]^{T} .
\end{aligned}
$$

Thereafter, the covariance matrix of the generated normal attributes is estimated through the simulation.

\subsection{Parameter estimation}

In order to calculate the previous equations, we should estimate parameters for multivariate normal distribution, in each treatment. To do this, regression can be used for each parameter when the hypothesis of its normality cannot be rejected. Eq. (11) shows multivariate normal distribution where $Y=\left[y_{1}, y_{2}, \ldots, y_{m}\right]$, $M=\left[\mu_{1}, \mu_{2}, \ldots, \mu_{m}\right]$, and $\sum=\left\lfloor\sigma_{y_{i} y_{j}}\right\rfloor(i=1,2, \ldots, m$; $j=1,2, \ldots, m)$ are response vector, mean vector, and covariance matrix, respectively:

$f\left(y_{1}, y_{2}, \ldots, y_{m}\right)=\frac{1}{(2 \pi)^{\frac{m}{2}}\left|\sum\right|^{\frac{1}{2}}} e^{\left(-\frac{1}{2}\left(Y-M^{\prime}\right) \sum^{-1}(Y-M)\right)}$,

where $\left|\sum\right|$ is determinant of covariance matrix and $(Y-M)^{\prime}$ is transpose of $(Y-M)$. As can be seen, we need to estimate mean and variance for each response and covariance for each couple of responses in each treatment. The least square estimators are as follows:

$$
\begin{aligned}
& \hat{\mu}_{i}=x \alpha_{i}, \quad i=1, \ldots, m, \\
& \log \left(\hat{\sigma}_{i}^{2}\right)=x \beta_{i}, \quad i=1, \ldots, m, \\
& \tanh ^{-1}\left(\hat{\rho}_{i j}\right)=x \gamma_{i j}, \quad 1 \leq i \leq j \leq m,
\end{aligned}
$$

where $x$ is a row vector of controllable variables and $\alpha_{i}, \beta_{j}$, and $\gamma_{i j}$ are the column vectors that their components determine the impact of associated variables.

To ensure positive values for variances, logarithmic model is used. On the other hand, as correlation coefficients should be between -1 and 1 , we use the inverse hyperbolic tangent transformation [19], which is defined as:

$$
\tan ^{-1}(\rho)=\frac{1}{2} \log \frac{(1+\rho)}{(1-\rho)} .
$$

Weighted optimality probability index can be cal-

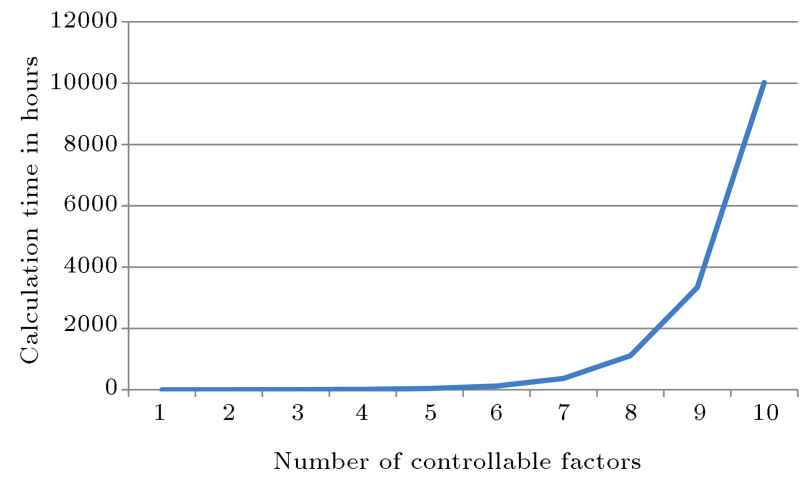

Figure 3. Computational time against the number of controllable factors.

culated for each possible treatment with respect to other treatments by estimating these parameters for responses corresponding to each treatment.

In the case of non-normal parameters, artificial neural network can be a good method to estimate parameters for any possible treatment. Note that the number of neurons can change with regard to each design.

\subsection{The proposed heuristic algorithm}

Calculation of WOPI or OOPI for all possible factorlevel combinations may be very time consuming, especially when we deal with sizeable number of responses and controllable factors.

For better comprehension, suppose that we want to calculate WOPI for some problems with different numbers of controllable factors. Figure 3 shows estimation of computational time to solve such problems on a notebook with an AMD E-350 processor and 4 gigabytes of RAM when all factors have 3 levels. To estimate the computational time, the algorithm was performed for few numbers of controllable factors and then, the computational time was estimated for large numbers of factors using the regression.

It can be seen that by increasing the number of factors, calculation time increases exponentially. So, a heuristic algorithm can be useful to reduce computational time. As another solution for the dominating time problem, we can use an artificial neural network. At first, we need to calculate WOPI for some treatments to train our network. Then, we can use the trained ANN instead of WOPI equation, but it is clear that this approach may cause non-negligible errors.

The proposed heuristic algorithm tries to find the most probable treatment in each iteration of probability calculation. By this approach, the solution space decreases in each iteration and, consequently, the number of calculations can be reduced. The proposed approach is described in Table 1 as pseudo-code.

Note that the proposed heuristic approach can be extended for problems with ordinal weights as well. 
Table 1. Pseudo-code of the proposed heuristic algorithm to find the best combination.

Transform all responses to STB type;

Find the estimation equations for the parameters of multivariate normal probability or train ANN to estimate responses;

Select a random factor-level combination $k$;

Calculate multivariate normal probability parameters for responses in combination $k$;

Calculate $\mathrm{WOPI}_{k}$;

Set $k^{*}=k$, and $\mathrm{WOPI}^{*}=\mathrm{WOPI}_{k}$;

Set the number of desired combinations to check $l$;

Select $l$ combinations with minimum $\mathrm{WPI}_{k, j}$ values and store in $J$;

repeat

for $k=J(1)$ to $J(l)$

Calculate multivariate normal probability parameters for responses in combination $k$;

Calculate $\mathrm{WOPI}_{k}$;

If $\mathrm{WOPI}_{k}>\mathrm{WOPI}^{*}$, set $k^{*}=k$ and $\mathrm{WOPI}^{*}=\mathrm{WOPI}_{k}$;

Next $k$;

Select $l$ combinations with minimum $\mathrm{WPI}_{k, j}$ value and store in $J$;

Until WOPI* does not change.

\section{Numerical examples}

In this section, two numerical examples from the previous articles are studied according to the proposed method and the results are compared with results of the reference articles. Then, a simulated numerical example with large number of factors is presented to show efficiency of the proposed heuristic algorithm.

\subsection{Example 1}

As the first example, consider an experimental design described by Chiao and Hamada [2]. The goal is to minimize the imbalance of a plastic wheel cover the component when we deal with two NTB quality characteristics: total weight $\left(Y_{1}\right)$ and the balance of the component $\left(Y_{2}\right)$. There are seven controllable factors with two levels which have effect on balance of the component. Table 2 shows the values of two correlated responses in the 8 runs.

The first step is using Johnson transformation [20] to change NTB responses into STB-types in order to use the proposed method as mentioned in Section 2. Eqs. (16) and (17) show the proper transformations for two responses and as can be seen, $Z_{1}$ and $Z_{2}$ are transformed into STB-type responses:

$$
\begin{aligned}
Z_{1}= & 0.387228+0.365089 \\
& \times \operatorname{Ln}\left(\frac{\left|Y_{1}-\mu_{1}\right|-0.0888751}{21.2924-\left|Y_{1}-\mu_{1}\right|}\right), \\
Z_{2}= & 0.122266+0.389592 \\
& \times \operatorname{Ln}\left(\frac{\left|Y_{2}-\mu_{2}\right|-0.0863844}{1.26185-\left|Y_{2}-\mu_{2}\right|}\right) .
\end{aligned}
$$

Now, the least square estimators can be written for multivariate distribution parameters using Eqs. (12)(15) as follows:

$$
\begin{aligned}
& \hat{\mu}_{1}=0.079-0.288 x_{4}+0.643 x_{5}-0.297 x_{7}, \\
& \hat{\mu}_{2}=-0.047+0.109 x_{3}+0.241 x_{1}+0.763 x_{5}-0.486 x_{7}, \\
& \log \left(\hat{\sigma}_{1}^{2}\right)=-1.060-0.390 x_{1}+0.202 x_{4}-0.267 x_{7}, \\
& \log \left(\hat{\sigma}_{2}^{2}\right)=-0.870-0.282 x_{2}, \\
& \tanh ^{-1}\left(\hat{\rho}_{1,2}\right)=-0.106+0.559 x_{1}-0.318 x_{5}-0.494 x_{7} .
\end{aligned}
$$

Table 3 shows three best factor-level combinations for this example. It can be concluded from Eqs. (18) that factor $F$ is not significant and can be ignored in the estimation of parameters. The optimal combination by the proposed method is $\left(-1,-1,-1,-1,-1,,_{-}, 1\right)$ with optimality probability index of $0.2326 \times 10^{-12}$, where an insignificant factor has been denoted by "_." To ensure that our proposed WOPI can find the true order of treatments, we can consider the same weights for responses and use Eq. (5) to calculate WOPI's. Moreover, the proportion of conformance, proposed by Chiao and Hamada [2], is calculated. It can be seen that the OPI and WOPI results are the same.

Now, suppose that the first response is important for us. Therefore, we deal with a multi-response optimization problem with ordinal response weights. As described before in section 2, to find the best treatment, we should calculate OOPI for each of them. Table 4 illustrates the first three treatments with corresponding ordinal optimality probability indices. 
Table 2. Responses values in Example 1.

\begin{tabular}{|c|c|c|c|c|c|c|c|c|c|c|c|c|c|}
\hline RUN & F1 & F2 & F3 & F4 & F5 & F6 & F7 & \multicolumn{6}{|c|}{ Replicates } \\
\hline \multirow[t]{2}{*}{1} & -1 & -1 & -1 & -1 & -1 & -1 & -1 & $Y_{1}$ & 711.9 & 713.4 & 712.3 & 712.4 & 711.9 \\
\hline & & & & & & & & $Y_{2}$ & 0.59 & 0.59 & 0.47 & 0.71 & 0.63 \\
\hline \multirow[t]{2}{*}{2} & -1 & -1 & -1 & 1 & 1 & 1 & 1 & $Y_{1}$ & 725 & 720.1 & 711.8 & 723.9 & 720.9 \\
\hline & & & & & & & & $Y_{2}$ & 0.7 & 0.91 & 1.13 & 0.79 & 0.78 \\
\hline \multirow[t]{2}{*}{3} & -1 & 1 & 1 & -1 & -1 & 1 & 1 & $Y_{1}$ & 711.6 & 711.7 & 711.3 & 712.1 & 711.7 \\
\hline & & & & & & & & $Y_{2}$ & 0.56 & 0.44 & 0.46 & 0.53 & 0.46 \\
\hline \multirow[t]{2}{*}{4} & -1 & 1 & 1 & 1 & 1 & -1 & -1 & $Y_{1}$ & 733.7 & 724.1 & 732 & 732.7 & 733.3 \\
\hline & & & & & & & & $Y_{2}$ & 1.5 & 1.55 & 1.38 & 1.45 & 1.45 \\
\hline \multirow[t]{2}{*}{5} & 1 & -1 & 1 & -1 & 1 & -1 & 1 & $Y_{1}$ & 725.4 & 721.6 & 722.6 & 723.1 & 721.1 \\
\hline & & & & & & & & $Y_{2}$ & 1.25 & 1.36 & 1.51 & 1.22 & 1.25 \\
\hline \multirow[t]{2}{*}{6} & 1 & -1 & 1 & 1 & -1 & 1 & -1 & $Y_{1}$ & 728.7 & 721.1 & 722.9 & 723 & 719.7 \\
\hline & & & & & & & & $Y_{2}$ & 1.17 & 0.97 & 0.98 & 0.97 & 0.73 \\
\hline \multirow[t]{2}{*}{7} & 1 & 1 & -1 & -1 & 1 & 1 & -1 & $Y_{1}$ & 726.6 & 731.4 & 731.4 & 729.6 & 731.3 \\
\hline & & & & & & & & $Y_{2}$ & 1.52 & 1.58 & 1.61 & 1.4 & 1.57 \\
\hline \multirow[t]{2}{*}{8} & 1 & 1 & -1 & 1 & -1 & -1 & 1 & $Y_{1}$ & 714.3 & 714.4 & 713.6 & 716.3 & 714.6 \\
\hline & & & & & & & & $Y_{2}$ & 0.57 & 0.51 & 0.44 & 0.44 & 0.56 \\
\hline
\end{tabular}

Table 3. Weighted optimality probability index versus POC for the best three treatments in Example 2.

\begin{tabular}{ccccccccc}
\hline $\mathbf{A}$ & $\mathbf{B}$ & $\mathbf{C}$ & $\mathbf{D}$ & $\mathbf{E}$ & $\mathbf{G}$ & $\mathbf{P O C}$ & $\mathbf{O P I}_{\boldsymbol{k}}$ & ${\text { Weighted } \mathbf{O P I}_{\boldsymbol{k}}}^{-1}$ \\
\hline-1 & -1 & -1 & -1 & -1 & 1 & 0.5332 & $3.4843 \times 10^{-13}$ & $3.4843 \times 10^{-13}$ \\
-1 & 1 & -1 & -1 & -1 & 1 & 0.71216 & $8.6260 \times 10^{-15}$ & $8.6160 \times 10^{-15}$ \\
-1 & -1 & 1 & -1 & -1 & 1 & 0.5332 & $1.1306 \times 10^{-18}$ & $1.1306 \times 10^{-18}$ \\
\hline
\end{tabular}

Table 4. The best three treatments with the considered ordinal weights in Example 1.

\begin{tabular}{ccccccc}
\hline $\mathbf{A}$ & $\mathbf{B}$ & $\mathbf{C}$ & $\mathbf{D}$ & $\mathbf{E}$ & $\mathbf{G}$ & $\mathbf{O O P I}_{\boldsymbol{k}}$ \\
\hline-1 & -1 & -1 & 1 & -1 & 1 & $3.1880 \times 10^{-173}$ \\
1 & 1 & -1 & -1 & -1 & 1 & $5.7665 \times 10^{-295}$ \\
-1 & 1 & -1 & -1 & -1 & 1 & $5.1807 \times 10^{-304}$ \\
\hline
\end{tabular}

To verify the efficiency of OOPI, WOPI with appropriate weights can be used. For this example, if we choose $w_{1}$ and $w_{2} 0.9$ and 0.1 , respectively, it can be seen that treatment $(-1,-1,-1,1,-1,-1)$ with a WOPI of $4.0041 \times 10^{-38}$ is the most probable one.

\subsection{Example 2}

Consider a simulated design which has nine controllable factors with three levels for each of them and two STB correlated responses. In such a problem, because of the number of factors and their levels, using a heuristic algorithm is necessary. Table 5 shows the calculated parameters for each treatment.
The least square estimators for multivariate normal distribution parameters can be found below:

$$
\begin{aligned}
& \hat{\mu}_{1}=6.518+0.494 x_{1}-0.271 x_{2}-1.758 x_{3} \\
& \quad+0.447 x_{3}^{2}+0.643 x_{5}+0.337 x_{8} x_{9}, \\
& \hat{\mu}_{2}=114.104+2.241 x_{4}-7.825 x_{6}-7.822 x_{8} \\
& \quad+4.587 x_{6} x_{8}, \\
& \log \left(\hat{\sigma}_{1}^{2}\right)=-2.011-0.054 x_{1} x_{2}+0.091 x_{7} x_{9} \\
& \quad+0.065 x_{8} x_{9}, \\
& \log \left(\hat{\sigma}_{2}^{2}\right)=1.701-0.216 x_{1}-0.223 x_{7}-0.077 x_{4} x_{6}, \\
& \tanh ^{-1}\left(\hat{\rho}_{1,2}\right)=-1.132+1.165 x_{1} x_{6}+0.125 x_{2} x_{3} .
\end{aligned}
$$

Solving such problem on a notebook with an AMD E350 dual-core processor and 4 gigabytes of RAM can 
Table 5. The calculated parameters in each treatment for Example 2.

\begin{tabular}{|c|c|c|c|c|c|c|c|c|c|c|c|c|c|c|}
\hline Treatment & $\mathbf{A}$ & $\mathbf{B}$ & $\mathrm{C}$ & $\mathbf{D}$ & $\mathbf{E}$ & $\mathbf{F}$ & $\mathrm{G}$ & $\mathbf{H}$ & I & $\mu_{1}$ & $\mu_{2}$ & $\sigma_{1}^{2}$ & $\sigma_{2}^{2}$ & $\rho_{(1,2)}$ \\
\hline 1 & 1 & 1 & 1 & 1 & 1 & 1 & 1 & 1 & 1 & 4.1470 & 102.2243 & 0.0108 & 15.1017 & -0.2968 \\
\hline 2 & 1 & 1 & 1 & 1 & 2 & 2 & 2 & 2 & 2 & 3.3581 & 112.8818 & 0.0068 & 1.9950 & -0.5470 \\
\hline 3 & 1 & 1 & 1 & 1 & 3 & 3 & 3 & 3 & 3 & 3.8452 & 114.4757 & 0.0042 & 6.3085 & 0.8369 \\
\hline 4 & 1 & 2 & 2 & 2 & 1 & 1 & 1 & 2 & 2 & 2.6830 & 103.8821 & 0.0079 & 8.8593 & -0.2996 \\
\hline 5 & 1 & 2 & 2 & 2 & 2 & 2 & 2 & 3 & 3 & 2.3656 & 95.9612 & 0.0020 & 1.8893 & -0.7966 \\
\hline 6 & 1 & 2 & 2 & 2 & 3 & 3 & 3 & 1 & 1 & 2.7403 & 109.2219 & 0.0028 & 3.4271 & -0.8795 \\
\hline 7 & 1 & 3 & 3 & 3 & 1 & 1 & 1 & 3 & 3 & 4.2255 & 102.5917 & 0.0204 & 15.2438 & 0.9620 \\
\hline 8 & 1 & 3 & 3 & 3 & 2 & 2 & 2 & 1 & 1 & 3.2540 & 106.0403 & 0.0034 & 7.8308 & 0.3466 \\
\hline 9 & 1 & 3 & 3 & 3 & 3 & 3 & 3 & 2 & 2 & 2.1211 & 100.5824 & 0.0054 & 2.8403 & 0.4179 \\
\hline 10 & 2 & 1 & 2 & 3 & 1 & 2 & 3 & 1 & 2 & 3.1943 & 103.1929 & 0.0040 & 1.3277 & -0.2117 \\
\hline 11 & 2 & 1 & 2 & 3 & 2 & 3 & 1 & 2 & 3 & 4.3310 & 109.2780 & 0.0192 & 2.1912 & 0.3644 \\
\hline 12 & 2 & 1 & 2 & 3 & 3 & 1 & 2 & 3 & 1 & 3.1498 & 106.6018 & 0.0019 & 7.9685 & 0.6525 \\
\hline 13 & 2 & 2 & 3 & 1 & 1 & 2 & 3 & 2 & 3 & 3.9268 & 112.3448 & 0.0011 & 1.3944 & -0.3144 \\
\hline 14 & 2 & 2 & 3 & 1 & 2 & 3 & 1 & 3 & 1 & 3.1411 & 108.5867 & 0.0073 & 9.6146 & -0.0389 \\
\hline 15 & 2 & 2 & 3 & 1 & 3 & 1 & 2 & 1 & 2 & 3.5400 & 98.00457 & 0.0045 & 3.2939 & -0.0311 \\
\hline 16 & 2 & 3 & 1 & 2 & 1 & 2 & 3 & 3 & 1 & 3.6162 & 107.0205 & 0.0036 & 0.6142 & -0.9760 \\
\hline 17 & 2 & 3 & 1 & 2 & 2 & 3 & 1 & 1 & 2 & 3.3618 & 97.19684 & 0.0013 & 12.3763 & 0.8491 \\
\hline 18 & 2 & 3 & 1 & 2 & 3 & 1 & 2 & 2 & 3 & 3.1058 & 106.5303 & 0.0060 & 6.0686 & -0.9593 \\
\hline 19 & 3 & 1 & 3 & 2 & 1 & 3 & 2 & 1 & 3 & 4.5658 & 102.2799 & 0.0122 & 1.0111 & 0.5382 \\
\hline 20 & 3 & 1 & 3 & 2 & 2 & 1 & 3 & 2 & 1 & 3.5561 & 101.0539 & 0.0040 & 6.8441 & 0.1881 \\
\hline 21 & 3 & 1 & 3 & 2 & 3 & 2 & 1 & 3 & 2 & 4.8375 & 101.3454 & 0.0075 & 3.4844 & -0.0213 \\
\hline 22 & 3 & 2 & 1 & 3 & 1 & 3 & 2 & 2 & 1 & 3.6075 & 103.2989 & 0.0051 & 0.0620 & 0.9199 \\
\hline 23 & 3 & 2 & 1 & 3 & 2 & 1 & 3 & 3 & 2 & 3.7011 & 96.95306 & 0.0043 & 3.2635 & -0.9215 \\
\hline 24 & 3 & 2 & 1 & 3 & 3 & 2 & 1 & 1 & 3 & 3.9105 & 104.8708 & 0.0003 & 5.3679 & 0.0167 \\
\hline 25 & 3 & 3 & 2 & 1 & 1 & 3 & 2 & 3 & 2 & 3.2120 & 107.8587 & 0.0044 & 17.8818 & 0.9324 \\
\hline 26 & 3 & 3 & 2 & 1 & 2 & 1 & 3 & 1 & 3 & 2.6008 & 103.7729 & 0.0004 & 0.7953 & 0.5587 \\
\hline 27 & 3 & 3 & 2 & 1 & 3 & 2 & 1 & 2 & 1 & 4.6149 & 102.5406 & 0.0093 & 1.3074 & -0.2479 \\
\hline
\end{tabular}

Table 6. Optimality probability for the best three treatments in Example 3.

\begin{tabular}{ccccccccccc}
\hline Rank & $\mathbf{A}$ & $\mathbf{B}$ & $\mathbf{C}$ & $\mathbf{D}$ & $\mathbf{F}$ & $\mathbf{G}$ & $\mathbf{H}$ & $\mathbf{I}$ & $\mathbf{O P I}_{\boldsymbol{k}}$ & $\mathbf{W O P I}_{\boldsymbol{k}}$ \\
\hline $\mathbf{1}$ & 1 & 3 & 2 & 1 & 3 & 3 & 1 & 1 & $4.8997 \times 10^{-128}$ & $4.8997 \times 10^{-128}$ \\
$\mathbf{2}$ & 1 & 3 & 2 & 1 & 3 & 2 & 1 & 1 & $1.0896 \times 10^{-144}$ & $1.0896 \times 10^{-144}$ \\
$\mathbf{3}$ & 1 & 3 & 2 & 1 & 3 & 1 & 1 & 1 & $3.1330 \times 10^{-169}$ & $3.1330 \times 10^{-169}$ \\
\hline
\end{tabular}

take much time, i.e. about 3340 hours. Therefore, it is better to use the proposed heuristic approach. By considering 0.5 as weight value for both responses, the proposed algorithm is coded in MATLAB. After about 3823 seconds as computational time, it shows that optimum combination is $\left(1,3,2,1,,_{-}, 3,3,1,1\right)$ with WOPI of $4.8997 \times 10^{-128}$ between 6561 possible combinations. The OPI values confirm our results. Table 6 shows optimality probability values for the best three combinations.

\subsection{Example 3}

Consider a $2^{3}$ full factorial design with three correlated STB type responses which are generated using NORTA method. Each treatment has four replicates per each response. Because there is no other possible treatment, we do not need to find estimators for parameters. Table 7 shows experimental design and response values for this example.

As expressed in Section 2, in such problems, first, we can use NORTA inverse method to transform multivariate exponential responses into normal ones and then calculate the optimality probability index. Based on the transformed response values, mean, variances and correlation coefficients can be calculated for each treatment. Table 8 shows multivariate normal parameters for each treatment.

After computing normal parameters, we can cal- 
Table 7. Experimental design with correlated exponential response in Example 3.

\begin{tabular}{|c|c|c|c|c|c|c|c|c|c|c|c|c|c|c|c|}
\hline \multirow{2}{*}{$\frac{\text { Treatment }}{1}$} & \multirow{2}{*}{$\frac{\mathbf{A}}{1}$} & \multirow{2}{*}{$\begin{array}{r}\mathbf{B} \\
-1\end{array}$} & \multirow{2}{*}{$\begin{array}{l}\mathrm{C} \\
-1\end{array}$} & \multicolumn{4}{|c|}{$Y_{1}$} & \multicolumn{4}{|c|}{$Y 2$} & \multicolumn{4}{|c|}{$Y 3$} \\
\hline & & & & 0.08 & 0.14 & 0.16 & 0.22 & 2.82 & 5.87 & 6.45 & 12.31 & 55.53 & 55.74 & 56.44 & 62.15 \\
\hline 2 & 1 & 1 & 1 & 0.35 & 0.39 & 0.40 & 0.42 & 19.34 & 20.10 & 23.61 & 23.95 & 175.70 & 177.25 & 223.08 & 226.15 \\
\hline 3 & -1 & -1 & 1 & 0.54 & 0.55 & 0.61 & 0.89 & 25.09 & 33.99 & 42.37 & 46.04 & 307.42 & 334.23 & 383.93 & 386.09 \\
\hline 4 & 1 & 1 & -1 & 1.00 & 1.10 & 1.17 & 1.19 & 50.06 & 56.37 & 56.60 & & 399.63 & 425.76 & & 487.42 \\
\hline 5 & 1 & -1 & 1 & 1.25 & 1.47 & 1.50 & 1.88 & 78.10 & 87.93 & 91.01 & 92.44 & 600.79 & 666.50 & 714.33 & 806.93 \\
\hline 6 & -1 & -1 & -1 & 2.22 & 2.31 & 2.64 & 2.76 & 100.75 & 111.85 & 116.95 & 119.34 & 1035.33 & 1076.38 & 1110.87 & 1240.80 \\
\hline 7 & -1 & 1 & -1 & 2.90 & 3.69 & 3.92 & 5.26 & 137.29 & 139.16 & 149.00 & 164.05 & 1482.25 & 1637.67 & 1689.20 & 1726.50 \\
\hline 8 & -1 & 1 & 1 & 5.40 & 6.66 & 8.50 & 16.15 & 233.06 & 233.16 & 255.92 & 311.97 & 1762.07 & 2062.43 & 3002.15 & 3641.25 \\
\hline
\end{tabular}

Table 8. Mean, variances, and correlation coefficients for normalized responses in Example 3.

\begin{tabular}{cccccccccc}
\hline Treatment & $\boldsymbol{\mu}_{\mathbf{1}}$ & $\boldsymbol{\sigma}_{\mathbf{1}}^{\mathbf{2}}$ & $\boldsymbol{\mu}_{\mathbf{2}}$ & $\boldsymbol{\sigma}_{\mathbf{2}}^{\mathbf{2}}$ & $\boldsymbol{\mu}_{\mathbf{3}}$ & $\boldsymbol{\sigma}_{\mathbf{3}}^{\mathbf{2}}$ & $\boldsymbol{\rho}_{(\mathbf{1}, \mathbf{2})}$ & $\boldsymbol{\rho}_{(\mathbf{1}, \mathbf{3})}$ & $\boldsymbol{\rho}_{(\mathbf{2}, \mathbf{3})}$ \\
\hline $\mathbf{1}$ & -1.33 & 0.24 & -1.81 & 0.12 & -1.37 & 0.13 & 0.98 & 0.87 & 0.75 \\
$\mathbf{2}$ & -0.75 & 0.01 & -1.28 & 0.19 & -0.70 & 0.01 & 0.90 & 0.96 & 0.80 \\
$\mathbf{3}$ & -0.39 & 0.03 & -0.56 & 0.00 & -0.40 & 0.00 & 0.84 & 0.66 & 0.92 \\
$\mathbf{4}$ & -0.04 & 0.00 & -0.42 & 0.01 & -0.29 & 0.00 & 0.80 & 0.88 & 0.96 \\
$\mathbf{5}$ & 0.27 & 0.00 & -0.11 & 0.01 & -0.01 & 0.01 & 0.85 & 0.93 & 0.96 \\
$\mathbf{6}$ & 0.45 & 0.00 & 0.28 & 0.03 & 0.16 & 0.00 & 0.98 & 0.93 & 0.96 \\
$\mathbf{7}$ & 0.80 & 0.13 & 0.70 & 0.02 & 0.93 & 0.04 & 0.76 & 0.87 & 0.94 \\
$\mathbf{8}$ & 2.08 & 0.51 & 1.67 & 0.23 & 1.69 & 0.27 & 0.96 & 0.94 & 0.95 \\
\hline
\end{tabular}

Table 9. OPI values for each treatment in Example 3.

\begin{tabular}{cc}
\hline Treatment & OPI \\
\hline $\mathbf{1}$ & 0.2887 \\
$\mathbf{2}$ & 0.0090 \\
$\mathbf{3}$ & $3.9543 \times 10^{-8}$ \\
$\mathbf{4}$ & $1.3368 \times 10^{-15}$ \\
$\mathbf{5}$ & $2.5850 \times 10^{-33}$ \\
$\mathbf{6}$ & $1.2941 \times 10^{-61}$ \\
$\mathbf{7}$ & $3.0134 \times 10^{-61}$ \\
$\mathbf{8}$ & $2.1035 \times 10^{-35}$ \\
\hline
\end{tabular}

culate optimality probability index for each treatment. Table 9 shows OPI for each treatment. It can be seen that the first treatment is the best factor-level combination by a probability of 0.2887 .

\subsection{A real case}

To study the applicability of the proposed method, consider a real case of air pollution in Tehran. Some of the important factors, including the number of taxies (NTX), the number of minibuses (NMB), the number of bus lines (NLB), the length of Lines for Bus Rapid Transportation in kilometer (LBRT), the length of metro lines in kilometer (LML), the Number of Metro Trains (NMT), and the Number of Metro Stations (NMS), were collected from 2009 to 2013 and are represented in Table 10 (from official webpage http://tmicto.tehran.ir). Moreover, three correlated air pollution indices including $\mathrm{CO}, \mathrm{O} 3$, and $\mathrm{NO}_{2}$ were gathered in the mentioned period and are illustrated in Table 11 (from official webpage http://air.tehran.ir).

The goal is to find the year with minimum polluted air by considering $w=\left[\begin{array}{lll}0.6 & 0.1 & 0.3\end{array}\right]$ as weights array. By applying the proposed method, it can be seen that 2013 is the best year with WOPI equal to $5.6864 \times 10^{-7}$. As another scenario, $r=\left[\begin{array}{lll}1 & 3 & 2\end{array}\right]$ is selected as order of responses. By calculating OOPI for all years, 2013 with OOPI $=0.1656$ is selected as the best year. Table 12 shows the WOPI and OOPI results for the years.

Table 10. The values of some important factors of air pollution indices in the different years.

\begin{tabular}{cccccccc}
\hline Year & NTX & NMB & NLB & LBRT & LML & NMT & NMS \\
\hline $\mathbf{2 0 0 9}$ & 70460 & 1356 & 359 & 62.1 & 106.2 & 95 & 64 \\
$\mathbf{2 0 1 0}$ & 72370 & 982 & 308 & 85 & 125 & 105 & 70 \\
$\mathbf{2 0 1 1}$ & 78857 & 1442 & 263 & 105 & 129 & 129 & 76 \\
$\mathbf{2 0 1 2}$ & 77949 & 1285 & 246 & 114.2 & 140 & 148 & 84 \\
$\mathbf{2 0 1 3}$ & 77949 & 1144 & 244 & 158.4 & 152 & 148 & 85 \\
\hline
\end{tabular}


Table 11. The mean, variance, and correlation coefficients of indices in different years.

\begin{tabular}{|c|c|c|c|c|c|c|c|c|c|}
\hline Year & $\mu_{C O}$ & $\sigma_{C O}^{2}$ & $\mu_{O 3}$ & $\sigma_{O 3}^{2}$ & $\mu_{N O 2}$ & $\sigma_{\mathrm{NO} 2}^{2}$ & $\rho_{(\mathrm{CO}, \mathrm{OB})}$ & $\rho_{(C O, N O 2)}$ & $\rho_{(\mathrm{NO} 2, \mathrm{O} 3)}$ \\
\hline 2009 & 48.022 & 525.639 & 48.315 & 1478.529 & 23.734 & 97.054 & -0.170 & 0.203 & 0.048 \\
\hline 2010 & 50.756 & 641.287 & 42.296 & 1259.854 & 46.476 & 1044.879 & -0.036 & 0.198 & -0.392 \\
\hline 2011 & 39.223 & 210.866 & 35.739 & 396.605 & 59.917 & 1096.366 & -0.010 & 0.225 & -0.085 \\
\hline 2012 & 39.330 & 273.022 & 37.189 & 433.455 & 55.074 & 616.104 & 0.004 & 0.258 & 0.040 \\
\hline 2013 & 36.732 & 206.044 & 41.417 & 339.423 & 54.636 & 705.961 & -0.105 & 0.369 & -0.253 \\
\hline
\end{tabular}

Table 12. WOPI for each year.

\begin{tabular}{ccc}
\hline Year & WOPI & OOPI \\
\hline $\mathbf{2 0 0 9}$ & $1.2139 \times 10^{-8}$ & 0.0261 \\
$\mathbf{2 0 1 0}$ & $3.8470 \times 10^{-9}$ & 0.0074 \\
$\mathbf{2 0 1 1}$ & $4.2432 \times 10^{-9}$ & 0.1284 \\
$\mathbf{2 0 1 2}$ & $4.1819 \times 10^{-9}$ & 0.1262 \\
$\mathbf{2 0 1 3}$ & $5.6864 \times 10^{-7}$ & 0.1656 \\
\hline
\end{tabular}

Although there are several nuisance factors such as rainfall, wind speed, and temperature inversion which have effect on the air pollution, it can be seen that increase in LML and LBRT as well as decrease in NMB could lead the indices to a better point in 2013. Therefore, based on the results, there is hope that continuing these transportation policies leads to decrease in the air pollution.

Note that there is not any proper alternative method to consider weights as well as correlation structure to compare the results. On the other hand, using methods which have independent viewpoints ignores the correlation structure and thus may lead to a false result.

\section{Conclusions}

Because of difficulty in considering correlated multiple responses in the optimization problems, there are a few studies in this field. However, such problem would be more difficult when the responses have not the same importance for us. In these cases, the significance of each response should be interpolated in the model as weights, either ordinal or cardinal. In this paper, we developed a probabilistic index to find the optimal treatment in an experimental design with normally distributed weighted correlated responses. Moreover, a transformation method from literature was considered to equip the proposed approach to solve the problems with non-normal correlated responses. To show the efficiency of the proposed optimality indices, three numerical examples were presented. In the first example, a comparison between OPI, WOPI, and OOPI showed that the results of indices could confirm each other. Moreover, a real case of air pollution in Tehran was studied to clarify the practical aspects of the proposed method. Other multivariate distributions, such as exponential, gamma, etc. can be considered for future studies.

\section{References}

1. Jeyapaul, R., Shahabudeen, P. and Krishnaiah, K. "Simultaneous optimization of multi-response problems in the Taguchi method using genetic algorithm", The International Journal of Advanced Manufacturing Technology, 30(9-10), pp. 870-878 (2005).

2. Chiao, C. and Hamada, M. "Analyzing experiments with correlated multiple responses", Journal of Quality Technology, 23(4), pp. 451-465 (2001).

3. Maghsoodloo, S. and Chang, C. "Quadratic loss functions and signal-to-noise ratios for a bivariate response", Journal of Manufacturing Systems, 20(1), pp. 1-12 (2001).

4. Maghsoodloo, S. and Huang, L. "Quality loss functions and performance measures for a mixed bivariate response", Journal of Manufacturing Systems, 20(2), pp. $73-88$ (2001).

5. Ozdemir, G. and Maghsoodloo, S. "Quadratic quality loss functions and signal-to-noise ratios for a trivariate response", Journal of Manufacturing Systems, 23(2), pp. 144-171 (2004).

6. Wu, F. "Optimization of correlated multiple quality characteristics using desirability function", Quality Engineering, 17(1), pp. 119-126 (2005).

7. Ko, Y., Kim, K. and Jun, C. "A new loss functionbased method for multiresponse optimization", Journal of Quality Technology, 37(1), pp. 50-59 (2005).

8. Antony, J. "Multi-response optimization in industrial experiments using Taguchi's quality loss function and principal component analysis", Quality and Reliability Engineering International, 16(1), pp. 3-8 (2000).

9. Liao, H. "Multi-response optimization using weighted principal component", The International Journal of Advanced Manufacturing Technology, 27(7-8), pp. 720725 (2006).

10. Datta, S., Nandi, G. and Bandyopadhyay, A. "Analyzing experiments with correlated multiple responses", Journal of Manufacturing Systems, 28(2-3), pp. 55-63 (2009).

11. Hejazi, T.H., Bashiri, M., Díaz-García, J.A. and Noghondarian, K. "Optimization of probabilistic multiple response surfaces", Applied Mathematical Modelling, 36, pp. 1275-1285 (2012). 
12. Noorossana, R., Tajbakhsh, S.D. and Saghaei, A. "An artificial neural network approach to multiple-response optimization", The International Journal of Advanced Manufacturing Technology, 40(11-12), pp. 1227-1238 (2009).

13. Salmasnia, A., Baradaran Kazemzadeh, R. and Mohajer Tabrizi, M. "A novel approach for optimization of correlated multiple responses based on desirability function and fuzzy logics", Neurocomputing, 91, pp. 56-66 (2012).

14. Bashiri, M. and Bakhtiarifar, M.H. "An optimality probability index for multiple correlated responses", Communications in Statistics-Theory and Methods, 43(20), pp. 4324-4336 (2014).

15. Niaki, S.T.A. and Abbasi, B. "Skewness reduction approach in multi-attribute process monitoring", Communications in Statistics-Theory and Methods, 36, pp. 2313-2325 (2007).

16. Riani, M. "Robust multivariate transformations to normality: Constructed variables and likelihood ratio tests", Statistical Methods and Applications, 13(2), pp. 179-196 (2004).

17. Niaki, S.T.A. and Abbasi, B. "Monitoring multiattribute processes based on NORTA inverse transformed vectors", Communications in Statistics-Theory and Methods, 38, pp. 964-979 (2009).

18. Cario, M.C. and Nelson, B.L. Modeling and Generating Random Vectors with Arbitrary Marginal Distributions and Correlation Matrix, Department of Industrial
Engineering and Management Sciences, Northwestern University, Technical Report (1997).

19. Rao, C.R., Linear Statistical Inference and Its Application, 2nd Ed.: John Wiley \& Sons (1973).

20. Choi, Y.-M., Polansky, A.M. and Mason, R.L. "Transforming non-normal data to normality in statistical process control", Journal of Quality Technology, 30(2), pp. 133-141 (1988).

\section{Biographies}

Mahdi Bashiri is an Associate Professor of Industrial Engineering at Shahed University. He holds a BS degree in Industrial Engineering from Iran University of Science and Technology, MS, and PhD degrees in this field from Tarbiat Modares University. $\mathrm{He}$ is recipient of the 2013 young national top scientist award from Academy of Sciences of the Islamic Republic of Iran. His research interests are facilities planning, metaheuristics, and multi-response optimization.

Mohammad Hasan Bakhtiarifar received his BS degree from Imam Hussein University, Tehran, Iran, and MS degree in Industrial Engineering from Shahed University in Tehran, Iran, where he is currently a PhD degree student in the same field. His research interests include design of experiments, multiple-response optimization, multivariate probabilities, and metaheuristic algorithms. 